\title{
Loss of GFI1 impairs pulmonary neuroendorine cell proliferation, but the neuroendocrine phenotype has limited impact on post-naphthalene airway repair
}

\author{
R Ilona Linnoila', Sandra Jensen-Taubman ${ }^{1}$, Avedis Kazanjian² and H Leighton Grimes ${ }^{2}$
}

Naphthalene exposure kills lung airway epithelial (Clara) cells, but is rapidly followed by Clara cell reconstitution coincident with proliferation of pulmonary neuroendocrine cells (PNEC). Although a role for mature PNEC in the reconstitution process has been excluded, the reconstituting progenitor cells have been suggested to enter a transient neuroendocrine (NE) differentiation phase before differentiating to Clara cells. Furthermore, these progenitors were suggested to be the target population for transformation to a NE tumor; small cell lung cancer (SCLC). Although the NE phenotype is central to SCLC oncogenesis, the relevance of NE differentiation to post naphthalene reconstitution remains to be determined. The Growth factor independent-1 (Gfi1) transcription factor is expressed in SCLC and is required for the NE differentiation of PNEC. Gfi ${ }^{-1-}$ mice display a 70\% reduction in airway cells that express NE markers, and cells that stain for NE markers show weak expression of some markers. Therefore, to determine the relevance of the NE phenotype to post-naphthalene reconstitution, we examined post-naphthalene reconstitution in $\mathrm{Gfi}^{-1-}$ mice. Our analyses indicate that the post-naphthalene regeneration process includes both airway epithelial proliferation and apoptosis. Gfi1 deletion lowered both airway epithelial proliferation and apoptosis; however, the post-naphthalene rate of increase in growth and apoptosis was not significantly different between $\mathrm{Gfi}^{-1-}$ mice and wild-type littermates. Moreover, the timing and extent of $\mathrm{CC} 10+$ cell regeneration was unaffected by Gfi1 deletion. These data suggest that neither Gfi1 nor the NE phenotype play a dominant role in the regeneration process. However, the few $\mathrm{Gfi}^{-1-}$ cells capable of NE differentiation show a significant reduction in post-naphthalene proliferation. The modest proliferation seen in $\mathrm{Gfi}^{-1-} \mathrm{NE}$ cells is consistent with the previously proposed role for Gfi1 in controlling neuroendocrine cancer growth.

Laboratory Investigation (2007) 87, 336-344. doi:10.1038/labinvest.3700527

KEYWORDS: Gfi1; naphthalene; neuroendocrine; PNEC

Pulmonary neuroendocrine cells (PNEC) are a rare population that reside in the bronchiolar epithelium and are characterized by their ability to secrete amines and peptides that act as growth and regulatory factors. ${ }^{1}$ PNEC are present as dispersed single cells or as clusters of cells referred to as neuroepithelial bodies (NEB). NEB are highly innervated and are believed to act as intrapulmonary chemoreceptors, sensitive to hypoxia and to perform several suggested 'corpuscular' functions such as nociceptive signal transmission and mechanosensation..$^{2-5}$ Pulmonary damage caused by chronic lung diseases, hypoxia, hyperoxia, extended exposure to environmental pollutants such as tobacco smoke, nitrosamines, ozone and naphthalene induces PNEC proliferation. ${ }^{6,7}$
Naphthalene is an aromatic hydrocarbon that is a constituent of tobacco smoke. ${ }^{8}$ Naphthalene specifically ablates the non-ciliated epithelial Clara cells that are most abundant in the bronchiolar epithelium. ${ }^{9}$ Cytochrome P450-2F2-isozyme oxidizes naphthalene in Clara cells into naphthalene $1 \mathrm{R}, 2 \mathrm{~S}$ epoxide, which is highly toxic to cells. ${ }^{10}$ Non-Clara cells of the airways lack this cytochrome P450 enzyme and therefore naphthalene administration to mice specifically ablates only Clara cells. Following naphthalene-induced ablation of Clara cells, the airway epithelium undergoes a dynamic reconstitution and repopulation in the injured airways. Clara cell reconstitution post-naphthalene induced ablation is associated with PNEC and NEB hyperplasia. ${ }^{6,11,12}$ Lung injury as a result of naphthalene administration

${ }^{1}$ Experimental Pathology Section, Cell and Cancer Biology Branch, CCR, NCl, NIH, Bethesda, MD, USA and ${ }^{2}$ Division of Immunobiology, Cincinnati Children's Hospital Medical Center, Cincinnati, OH, USA

Correspondence: Professor HL Grimes, Division of Immunobiology-MLC 7038, Cincinnati Children's Hospital Medical Center, 3333 Burnet Avenue, Room 5564, Cincinnati, Cincinnati, OH 45229-3039, USA. E-mail: lee.grimes@cchmc.org 
provides a model to study the dynamics of reconstitution and to characterize progenitor cells responsible for the repair of injured lungs. ${ }^{6,9,11-15}$

The naphthalene injury and repair model has been utilized to define progenitors responsible for injury recovery and reconstitution of depleted epithelial cells. ${ }^{11,12,16-20}$ The NEB hyperplasia associated with naphthalene injury suggests a possible role for the PNEC in the reconstitution process. ${ }^{11,12,16}$ However, more recent data indicate that PNEC themselves are incapable of Clara cell reconstitution of the airways. ${ }^{16,17,19}$ It has been suggested that the NEB microenvironment serves as a reservoir for a variant Clara cell that (1) lacks cytochrome P450-2F2-isozyme activity rendering it resistant to naphthalene toxicity and (2) may be capable of proliferating and repopulating the injured lungs. ${ }^{16,17}$ Several other progenitors and niches have been described as potential contributors to the post-naphthalene reconstitution of Clara cells. ${ }^{18-21}$ For example, in the trachea and larger airways basal cells have the ability to proliferate and might contribute to the recovery process after naphthalene-induced Clara cell ablation. ${ }^{19,21}$ In addition, distinct from the NEB microenvironment, naphthalene-resistant Clara cells at the terminal bronchioalveolar duct junctions represent another potential contributor to the restoration of the injured epithelium. ${ }^{18}$ More recently, a progenitor at the bronchioalveolar duct junction was characterized, which was hypothesized to repopulate both damaged Clara and alveolar cells. ${ }^{20}$ The presence of several putative progenitors indicates the complexity of the process.

Extensive activation of the hedgehog ( $\mathrm{Hh}$ ) pathway has been suggested to immediately precede both neuroendocrine (NE) differentiation during post-naphthalene reconstitution, and normal differentiation of pulmonary NE precursor cells. ${ }^{22}$ Watkins $e t a l^{22}$ proposed that some types of small cell lung cancer (SCLC) may recapitulate the NE differentiation phase of Hh-dependent airway epithelial reconstitution. Thus, cells similar to the post naphthalene reconstituting cells were suggested to be the target population for transformation to SCLC. Although the NE phenotype is central to SCLC oncogenesis, the relevance of $\mathrm{NE}$ differentiation to postnaphthalene reconstitution remains to be determined.

The Growth factor independent-1 (Gfil) transcriptional repressor was first described as a target of insertion mutagenesis in Moloney murine virus induced leukemias. ${ }^{23-26}$ Senseless, the Drosophila orthologue of Gfil, is essential for fly sensory organ development. Senseless functions as a downstream effector of proneural basic helix-loop-helix (bHLH) proteins in antagonism to Notch. ${ }^{27}$ Similar interactions between Gfil and proneural bHLH exist in the mammalian sensory organs such as the ear and the retina. ${ }^{27-30}$ We recently reported the expression of Gfil and the proneural bHLH factor Achaete scute homolog-1 (mASH1) in both embryonic and adult mouse PNEC, and NE tumor cell lines. ${ }^{28,31}$ Indeed, although Gfil deletion in mouse PNEC did not affect mASH1 expression, lack of Gfil resulted in dra- matically reduced expression of NE markers such as calcitonin gene-related peptide (CGRP) and synaptophysin (SYN). Similarly, a strong correlation exists between expression of Gfil, ASH1 and NE markers in primary human lung tumors. ${ }^{31}$ Thus, Gfil plays an important role in the differentiation and proper maturation of PNEC, and lack of Gfi1 significantly decreases the NE phenotype of airway cells. ${ }^{31}$

Given the proposed link between NE differentiation in post naphthalene repair and SCLC oncogenesis, the expression of Gfil in SCLC, and the significant impairment of NE marker expression in $G f i 1^{-/-}$mice, we tested whether $G f i 1^{-/-}$ NE cells respond to post-naphthalene cues, and whether the reduced NE environment in Gfil null mice affects repair. Although deletion of Gfil reduced NE differentiation, NE cell proliferation, and airway epithelial proliferation and apoptosis, the post-naphthalene rate of increase in proliferation and apoptosis was not de-regulated. Thus, the regeneration process was not visibly affected. Our data suggest that neither Gfil nor the NE phenotype play a dominant role in the regeneration process.

\section{MATERIALS AND METHODS}

\section{Animals and Treatment}

Wild-type (wt) and $\mathrm{Gfi1}^{-1-}$ mice on a C57Bl/6 background were used at 2-months of age. Groups of wt and Gf1 null littermates were utilized throughout the study. Naphthalene (Sigma, St Louis, MO, USA) was dissolved in corn oil and administered intraperitoneally as described previously. ${ }^{11} \mathrm{Wt}$ and $\mathrm{Gfi}^{-1-}$ control mice were injected similarly with corn oil alone. The mice were killed 3 or 5 days following treatment. Two hours before death, bromo-deoxyuridine (BrdU) (Sigma) was administered. All control and experimental animals received BrdU. BrdU was dissolved in water at $10 \mathrm{mg} / \mathrm{ml}$ and administered intraperitoneally at $10 \mu \mathrm{g} / \mathrm{g}$ body weight.

\section{Lung Fixation and Tissue Collection}

Mice were killed, and the lungs were harvested after formalin fixation as previously described with minor modifications. ${ }^{11}$ Right lung lobes were fixed by inflation at $10 \mathrm{~cm} \mathrm{H}_{2} \mathrm{O}$ with neutral-buffered Formalin instilled through a tracheal cannula. ${ }^{11}$ The harvested lungs were further fixed in $10 \%$ neutral-buffered formalin (Surgipath, Richmond, IL, USA) overnight at room temperature. The $10 \%$ neutral buffered formalin was replaced with 70\% ethanol and the tissues were embedded into paraffin.

\section{Immunohistochemistry}

Paraffin-embedded tissue sections were deparaffinized, hydrated and stained using the Vectastain $\mathrm{ABC}$ kit (Vector Laboratories, Burlingame, CA, USA) following the vendor's instructions with modifications as described. ${ }^{32}$ Rabbit antiCGRP (1:3000, Amersham Biosciences, Piscataway, NJ, USA) and rabbit anti SYN (1:100, Zymed, San Fransisco, CA, USA) antibodies were employed to evaluate NE differentiation, 
whereas rabbit anti-Clara cell $10 \mathrm{kDa}$ secretory protein (CC10) (1:100 000, Dr Franco DeMayo, Baylor School of Medicine, Houston, TX, USA) was used to identify the major secretory product of the Clara cell, a signature of epithelial cell differentiation within the conducting airways. A rat antiBrdU antibody (1:300, Accurate Chemical, Westbury, NY, USA) was used to detect BrdU incorporation in cells undergoing proliferation. Following immunohistochemical staining NE differentiation was quantified by counting the positive foci composed of solitary PNEC and NEBs containing CGRP or SYN in airways. NE expression was computed for each animal by dividing the number of positive NE foci (solitary PNEC or NEBs) by the total number of airways analyzed (Foci/Airway Ratio or F/A). CC10 immunostaining pattern was assessed using a staining index as described previously, ${ }^{33}$ which combines the intensity and distribution of positive cells.

\section{Morphometric Analysis of Proliferation and Apoptosis}

Apoptotic index was determined by the TUNEL assay using the Apoptag Plus Peroxidase in situ Detection Kit (Chemicon International, Inc., Temecula, CA, USA). according to the vendor's instructions. Quantification of BrdU incorporation and TUNEL staining was assessed using MetaMorph imaging software (Universal Imaging, Westchester, PA, USA) with a $\times 20$ objective (Nikon Eclipse E400 Microscope). A labeling index (LI) for airway epithelial cells was derived by dividing the total number of positive nuclei by 1000 , the total number of cells analyzed per animal.

In addition to the overall labeling index, terminal bronchioles were defined as described previously, ${ }^{18}$ and cells located $200 \mu \mathrm{m}$ from the bronchioloalveolar junction were analyzed. To determine the rate of $\mathrm{BrdU}$ incorporation in NE cells adjacent sections placed on the same slide were stained for CGRP and BrdU and the percentage of labeled PNEC and NEBs was established.

\section{Statistical Analysis}

Mean values for all parameters were compared with control values using the Mann-Whitney Rank sum test unless indicated otherwise. Statistical significance for all comparisons was acknowledged when $P$-values were $<0.05$. The comparison of the fold-change between wt and $\mathrm{Gfil}^{-/-}$were determined using two-way ANOVA. The factors were treatment (naphthalene vs oil) and type (wt $v s \mathrm{Gfil}^{-1-}$ mice). A variance stabilizing transform function was used for the percentages (the arcsin square root). With this model, a significant interaction would mean that the effect of treatment (naphthalene $v s$ oil) will be different depending on type (wt vs $G f i 1^{-1-}$ ). This model does not test whether the ratios are different between the two types, but tests whether the magnitude of the effect measured on the transformed scale (arcsin square root of percent) are different between the two types. The SPSS statistical software (SPSS Inc. Chicago, IL) was used for performing the two-way ANOVA analysis.

\section{RESULTS}

\section{Differentiated $\mathrm{Gfi1}^{-/}$PNEC Proliferate Poorly, but} Respond Normally to Naphthalene-Induced Lung Injury We have determined previously that Gfil is expressed in embryonic and adult PNEC and that lack of Gfil expression resulted in reduced numbers of of CGRP and SYN expressing cells. ${ }^{28,31}$ In contrast, the number of Ash1 expressing cells in $\mathrm{Gfil}^{-1-}$ mice remained the same. ${ }^{28,31}$ As Ash1 is critically required for PNEC development, ${ }^{34}$ Gfil might be required for maturation of presumptive PNEC. ${ }^{28,31}$ Interestingly, some PNEC were able to differentiate in the absence of Gfil, but could only weakly express CGRP and SYN. To determine the relevance of the NE phenotype to the post-naphthalene reconstitution process, we determined CGRP and SYN expression in naphthalene injured airway epithelium of wt and $\mathrm{Gfil}^{-1-}$ mice 3 or 5 days following naphthalene administration. These time points are likely to be most informative for assessing NE differentiation. ${ }^{6}, 12$ Immunohistochemical detection of CGRP and SYN in oil-administered mice was used as a control (Figure 1a). Following naphthalene injury the number of PNEC increased. ${ }^{6,12}$ In our experiments, control mouse CGRP and SYN-positive cells within bronchioles increased in numbers, peaking 5 days following naphthalene administration (Figure 1a). ${ }^{6,12}$ Similar immunohistochemical analysis of $\mathrm{Gfi1}^{-/-}$mice show that the 2-3-fold increase in numbers of CGRP and SYN positive foci in wt $v s \mathrm{Giil}^{-/-}$mice was not statistically different (compare Figures $1 \mathrm{a}$ and $\mathrm{b}$ ). Thus, whereas few PNEC differentiate without Gfil, those that do are capable of responding to naphthalene-induced reconstitution signals.

Figure 1 wt and $\mathrm{Gfi}^{-1-}$ mice have a comparable fold increase in the foci with NE differentiation during lung reconstitution, but decreased proliferation rate. (a) Representative CGRP immunohistochemical detection of wt airway epithelium 5 days following oil administration (O5) and serial sections of CGRP and SYN staining 5 days after naphthalene administration (N5) (Scale bar, $50 \mu \mathrm{m}$ ). (b) CGRP staining of Gfi ${ }^{-/-}$mice airways epithelium 5 days following oil administration (O5) and serial sections of CGRP and SYN staining 5 days following naphthalene administration (N5). Note strong CGRP immunoreactivity in NEBs of wt animals compared with weaker staining in small select (2-3) NE cells in GFI1KO mice 5 days after naphthalene treatment (Scale bar, $50 \mu$ m). (c) Quantification of CGRP immunohistochemical staining data of both oil and naphthalene administered wt (WT) and Gfi1 ${ }^{-/-}$mice (KO). Lungs administered with oil and analyzed 3 or 5 days post-treatment were combined (3-5). The lungs of naphthalene-administered mice are presented separately for each time point. Data represent the number of NE foci in the airways (mean \pm standard deviation). Two-way ANOVA statistical analysis was used to compare the fold changes after 3 and 5 days of naphthalene treatment between WT and KO. (d) Quantification of BrdU incorporation rate in individual CPRP-containing NE cells of both oil and naphthalene administered wt and $\mathrm{Gfi}^{-/-}$(KO) mice. Lungs administered with oil and analyzed 3 or 5 days after administration were combined (3-5). The lungs of naphthalene-administered mice are presented separately for each time point. 

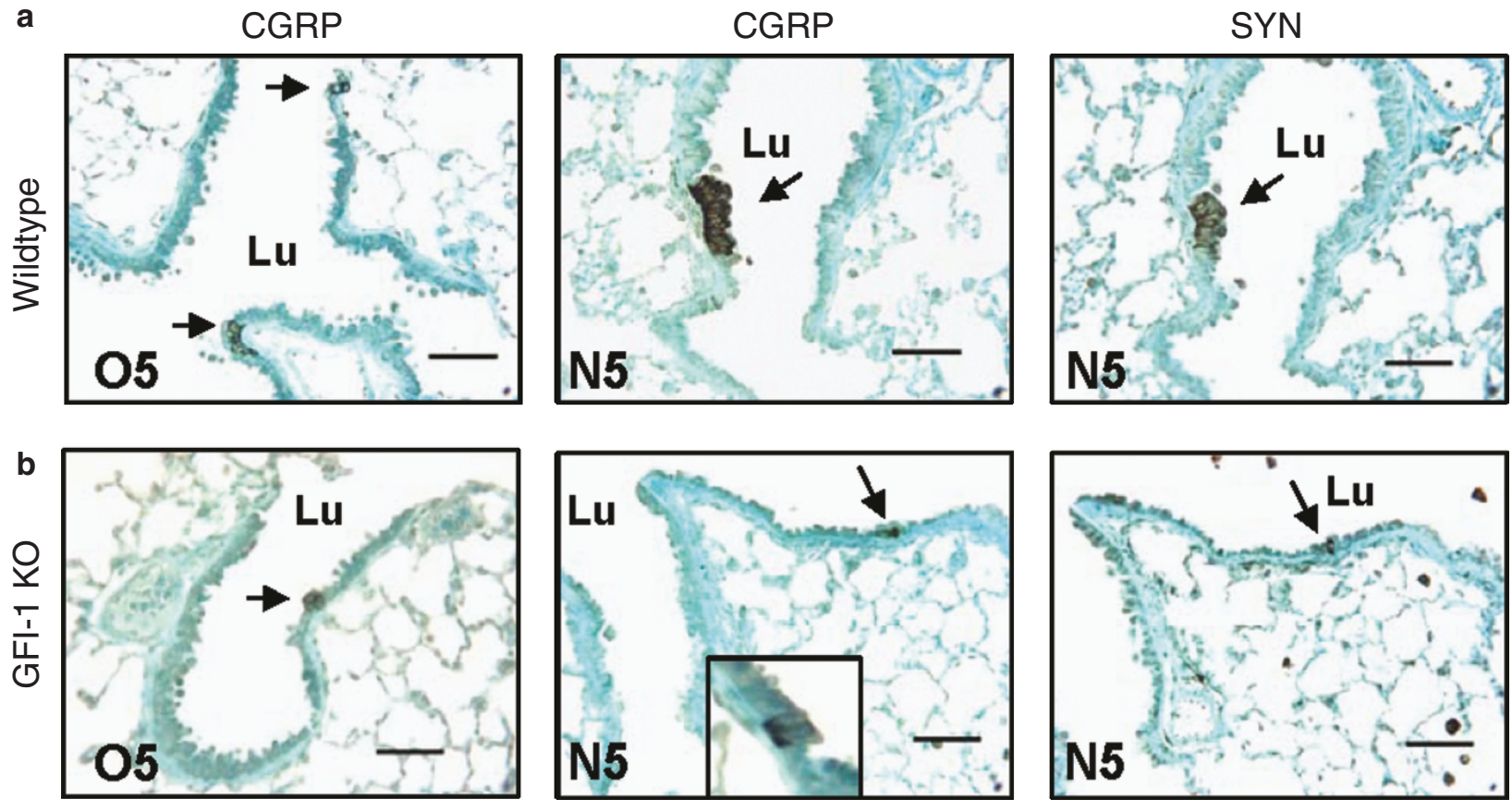

C

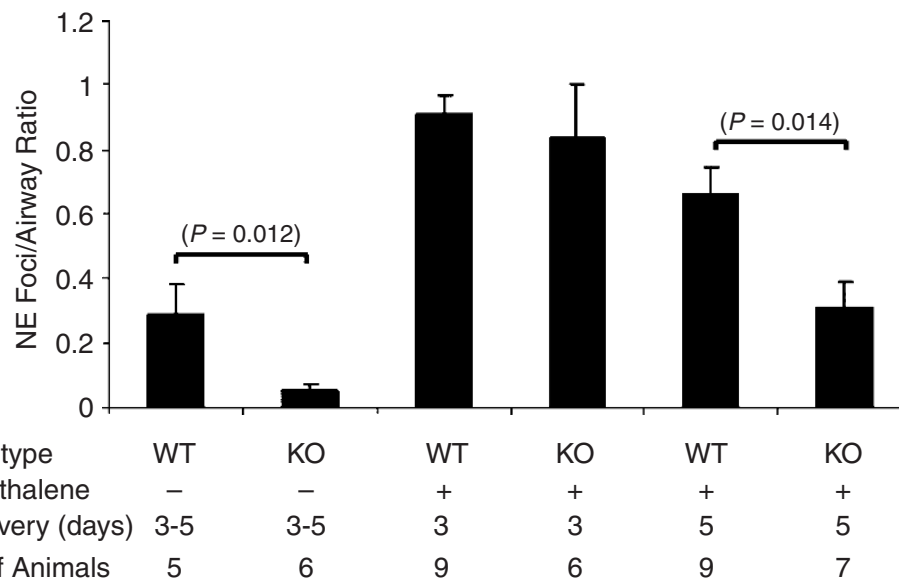

No. of Animals

d

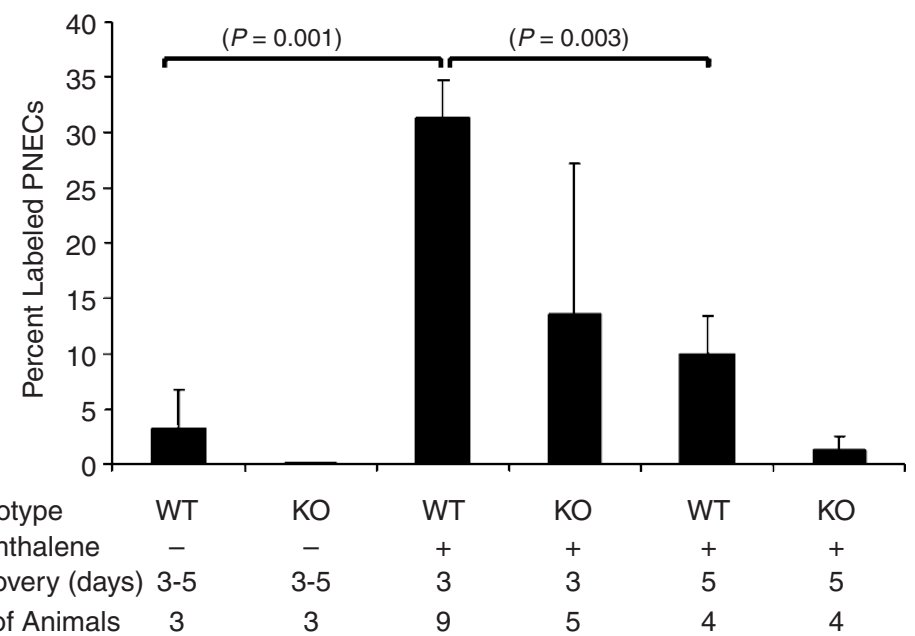


The increase in NE cells post-naphthalene exposure does not rescue the impact of $\mathrm{Gfil}^{-1-}$ on PNEC development. As expected, immunohistochemical staining showed a significant decrease of CGRP staining in oil-administered $\mathrm{Gfil}^{-/-}$ mice than in the oil-administered wt mice $(P=0.012)$ (Figure 1c). A similar decrease was observed in SYN expression in oil-administered $\mathrm{Gfil}^{-/-}$mice than in the oil-administered wt mice. In agreement with previous observations of postnaphthalene reconstitution, ${ }^{6,12}$ a significant increase in CGRP expressing NE cell foci within bronchiolar epithelium was observed in wt mice $3(P=0.016)$ and $5(P=0.032)$ days following naphthalene administration compared with oiltreated animals (data not shown). A similar increase in CGRP-containing foci was seen in $G f i 1^{-/}$mice $3(P=0.002)$ and $5(P=0.019)$ days following naphthalene administration compared with oil-administered animals (data not shown). However, the post-naphthalene environment did not rescue $\mathrm{Gfil}^{-1-}$ defects, because when $\mathrm{Gfil}^{-/-}$mice were compared with wt mice 5 days following naphthalene administration, there was still a significant reduction in CGRP expressing NE foci $(P=0.014)$ in the $\mathrm{Gfil}^{-/-}$animals (Figure 1c).

Similar to the increase in CGRP-positive NE foci following naphthalene administration, an increase in the wt SYN containing foci within bronchioli was observed compared with oil-treated mice. The increase in wt SYN-positive foci was significant 5 days following naphthalene administration compared with oil-treated mice $(P=0.049)$ (data not shown). Gfi1 ${ }^{-1-}$ mice also showed a significant increase in SYN-positive cells 5 days after naphthalene treatment when compared with oil-treated $G f_{i 1} 1^{-/}$animals $(P=0.005)$ (data not shown). As expected from the differences in PNEC induced by Gfil loss, comparison of SYN staining in $\mathrm{Gfil}^{-1-}$ mice and wt mice 5 days following naphthalene administration showed a significant difference between the two; $G \mathrm{fi1}^{-/-}$mice had less SYN-positive foci compared with wt mice $(P=0.009)$ (data not shown).

A two-way ANOVA analysis indicated that whereas the increase in the number of CGRP or SYN expressing foci in each group (wt or $\mathrm{Gfi1}^{-/-}$) is significantly different after naphthalene administration, there is no evidence that either wt or $\mathrm{Gfi1}^{-/-}$PNEC respond differently to naphthalene administration. Thus PNEC, which have managed to differentiate without Gfil respond to naphthalene treatment in a similar manner to normal PNEC.
To examine further the impact of Gfil loss on NE differentiation we analyzed BrdU incorporation within CGRP + cells (PNEC and NEBs). We first counted BrdU + NEBs. In wt mice approximately one out of five NEBs (18\%) initially revealed one or more BrdU-labeled cells, increasing to two out of three NEBs (69\%) 3 to 5 days post-naphthalene (data not shown). Next, we evaluated each individual CGRP + cells for BrdU staining. The post-naphthalene increase in $\mathrm{BrdU}+\mathrm{CGRP}+$ cells was significant $(P=0.001)$, but transient (Figure 1d). In contrast, BrdU + NEBs were rarely seen in $\mathrm{Gfil}^{-/-}$mice, and after exposure to naphthalene only one out of three $(35 \%)$ NEBs were also BrdU + (data not shown). Similar to wt littermates, the post-naphthalene increase in individual $\mathrm{Gfil}^{-/-} \mathrm{BrdU}+\mathrm{CGRP}+$ cells was transient (Figure 1d). Notably, the 3-day post-napthalene BrdU staining in $G \mathrm{fi1}^{-/-}$airways was highly variable, and prevented the increase in BrdU $+\mathrm{NE}$ cells from reaching statistical significance. Yet, the number of BrdU + CGRP + cells was lower in Gfil KO mice than wt littermates in both oil-treated controls, and at either 3 or 5 days after naphthalene $(P=0.05)$ (Figure 1d). The decreased BrdU labeling in $\mathrm{Gfil}^{-/-}$CGRP + cells is consistent with a role for Gfil in mediating proliferation of NE cells. However, the $G f i 1^{-1-}$ impairment of NE cell proliferation was not impacted by naphthalene treatment, as the naphthalene-induced rate of increase of the BrdU + CGRP + cells was not statistically different between wt and $G f i 1^{-1-}$ littermates $(P=0.8)$. In other words, $\mathrm{Gfil}^{-1-}$ airway epithelial cells that are capable of NE differentiation proliferate less well than Gfil expressing wt NE cells, but the naphthalene-induced rate of increase in the number of such cells was not impacted by loss of Gfil.

\section{Gfi1 Loss Decreases Post-Naphthalene Proliferation in Airway Epithelium}

BrdU incorporation was also utilized to assess the role of Gfil loss on proliferation in the airway epithelium 3 and 5 days following naphthalene injury (Figure 2). As expected, there was a significant increase in the overall epithelial cell proliferation rate in wt animals $(P<0.001)$ whereas the response in $\mathrm{Gfil}^{-1-}$ mice was attenuated $(P=0.058 ; 27$ fold $v s$ sevenfold, respectively) (Figure 2e). To characterize further post-naphthalene cellular dynamics, we focused on the terminal bronchioli, because this region comprises a putative regenerating zone harboring a stem cell niche. ${ }^{18,20}$

Figure 2 Airway epithelial cell proliferation and apoptosis is reduced in $\mathrm{Gfi}^{-/-}$mice, but the post-naphthalene-induced rate of increase in proliferation and apoptosis is not significantly altered in terminal bronchiolar epithelium. (a) Representative BrdU staining of wt bronchiolar airway epithelium 5 days following oil treatment (O5) (Scale bar, $50 \mu \mathrm{m}$ ). (b) BrdU staining of $\mathrm{Gfi}^{-1-}$ airways 5 days after oil administration (Scale bar, $50 \mu \mathrm{m}$ ). (c) wt airway BrdU staining 3 days following naphthalene administration (N3) (Scale bar, $50 \mu \mathrm{m}$ ). (d) Airway BrdU staining of Gfi ${ }^{-/-}$lungs 3 days post-naphthalene administration (Scale bar, $50 \mu \mathrm{m}$ ). (e) Quantification of the immunohistochemical data of BrdU staining of both wt and Gfi ${ }^{-/-}$mice (KO) 3 and 5 days following oil or naphthalene administration (3-5 represent combined data for both days). Data represent the percentage of BrdU-positive cells in all airways (black bars) and in the most distal $200 \mu \mathrm{m}$ of terminal bronchiolar epithelium (white bars; mean \pm standard error of mean). (f) Quantification of the immunohistochemical data of TUNEL staining of both wt and Gfi $^{-1}$ - mice (KO) 3 and 5 days following oil or naphthalene administration (3-5 represent combined data for both days). Data represent the percentage of TUNEL-positive cells in the most distal $200 \mu \mathrm{m}$ of terminal bronchiolar epithelium (mean \pm standard error of mean). 

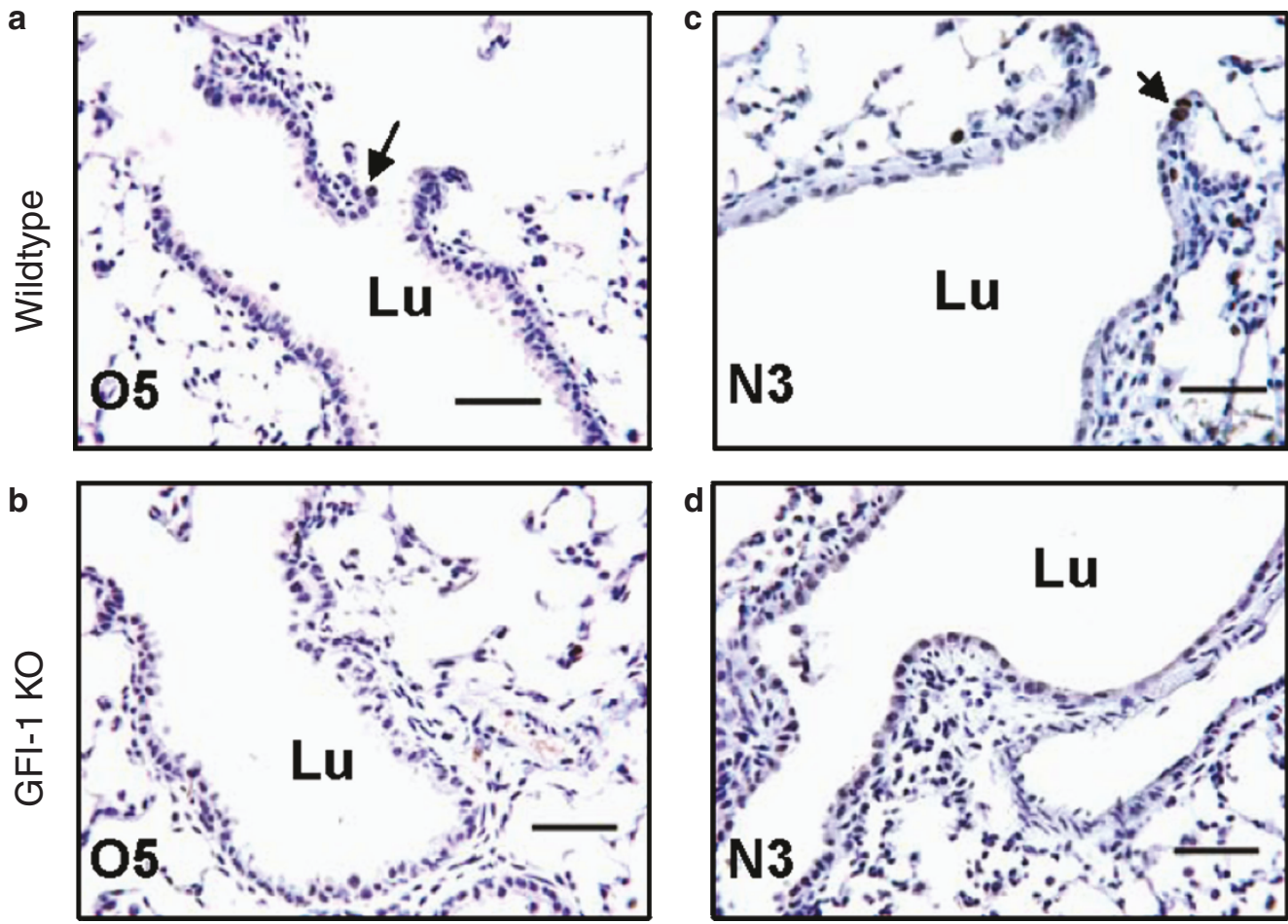

e
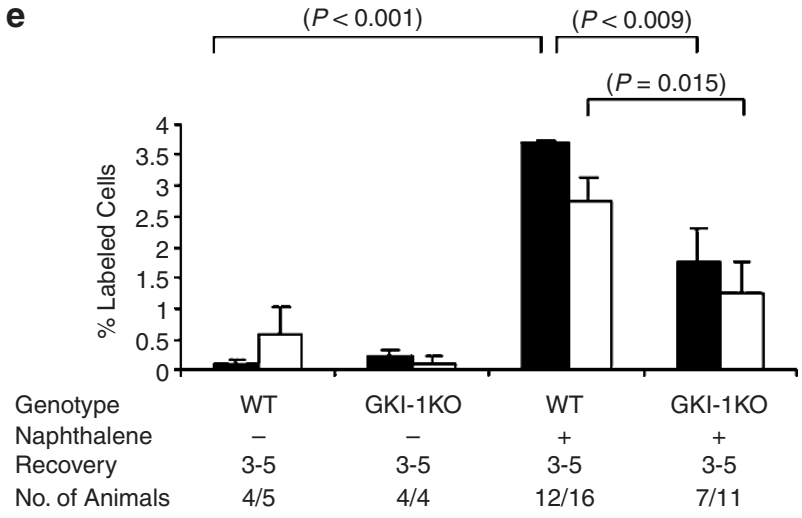

Black columns $=$ Proximal and distal airways,

White columns $=200$ um of terminal airway epithelium

$\mathbf{f}$

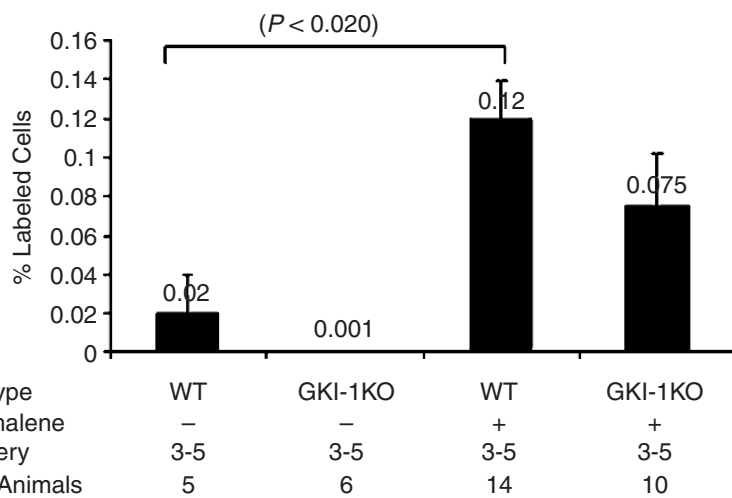


Immunohistochemical analysis of oil-administered wt or $\mathrm{Gfi1}^{-1-}$ mice revealed few cells actively proliferating in terminal bronchioli (Figures $2 \mathrm{a}$ and $\mathrm{b}$ ). Three days following naphthalene administration fewer cells were BrdU-positive in $\mathrm{Gfil}^{-1-}$ mice compared with wt mice (Figure $2 \mathrm{c}$ and d). Notably, the observed induction of post-naphthalene proliferation in terminal bronchioli was not significantly different between $\mathrm{Gfil}^{-/-}$and wt littermates. Similar to Gfil effects on $\mathrm{NE}$ cell proliferation, the $\mathrm{Gfil}^{-/-}$decrease in BrdU staining in terminal bronchioli was not reversed by naphthalene. Thus, whereas Gfil deletion decreases airway epithelial proliferation, the significance of Gfil deletion upon post-naphthalene proliferation is restricted to the proximal and distal airways.

We next examined apoptosis in terminal bronchioli. After naphthalene administration, there was a statistically significant increase in apoptotic (TUNEL +$)$ cells both in wt and $G f i 1^{-1-}$ mouse airway epithelial cells $(P<0.02$ and $P<0.05$, respectively; Welch $T$-test) (Figure $2 \mathrm{f})$. However, the difference between $G f i 1^{-1-}$ and wt littermates in the postnaphthalene rate of increase in apoptosis was not statistically significant $(P<0.1$; Welch $T$-test $)$ (Figure $2 \mathrm{f})$. In conclusion, our analyses have uncovered $\mathrm{Gfil}^{-1-}$ defects in airway epithelia and NE cell biology, but ANOVA statistical analyses indicated that these defects do not interact with naphthalene treatment.

\section{Clara Cell Lung Reconstitution is Efficient in $\mathrm{Gfi1}^{-/-}$Mice} Given a lack of statistical correlation between $\mathrm{Gfi}^{-/-}$defects and naphthalene treatment, we next examined whether lack of Gfil expression in murine lungs has an effect on Clara cell reconstitution. First, CC10 immunohistochemical staining of the distal airways 3 days following oil administration showed no initial difference between $\mathrm{Gfi1}^{-1-}$ and wt mice (Figures 3a and b). Moreover, Clara cells in the $\mathrm{Gfil}^{-1-}$ airway are ablated similarly to wt littermate airway cells (Figure 3c). Finally, similar to wt mice, Clara cells in $\mathrm{Gfi1}^{-/-}$lungs were fully reconstituted by 5 days following naphthalene administration (Figure 3d).

Quantification of CC10-positive cells showed no difference in staining between wt and $\mathrm{Gfil}^{-1-}$ mice following oil treatment. A significant decrease was observed in both $\mathrm{Gfil}^{-1-}$ and wt mice 3 days following naphthalene administration compared with oil-treated animals $(P<0.001)$ (Figure $3 e$ ), confirming successful ablation with naphthalene. Wt and $\mathrm{Gfi1}^{-/-}$mice showed very similar Clara cell reconstitution with no difference observed between the two, 5 days following naphthalene treatment (Figure 3e).

\section{DISCUSSION}

Discerning the mechanisms that are active during post-injury lung regeneration is crucial for decreasing the impact of injury from environmental insults or disease. Functional knockouts that delete cells expressing the CC10 marker were used previously to examine the process of naphthalene-in- duced lung injury and regeneration. ${ }^{17}$ However, no previous studies have examined the response to naphthalene-induced injury in a model with impaired expression of NE differentiation or impaired PNEC development.

We have reported previously the requirement of Gfil for the proper differentiation of PNEC during development. ${ }^{31}$ Others have implied that reconstitution following naphthalene exposure involves a wave of NE differentiation. ${ }^{6,22}$ The present study used the naphthalene-induced lung injury model to evaluate the functional consequences of impaired NE phenotype upon Clara cell reconstitution. ${ }^{35}$

Following naphthalene injury, PNEC undergo proliferation with concomitant increase in NE marker expression. ${ }^{6} \mathrm{Al}-$ though the levels of NE cells were lower in $\mathrm{Gfil}^{-1-}$ mice, the post-naphthalene rate of change between the wt and $\mathrm{Gfil}^{-/-}$ mice were similar. PNEC proliferation represents only a small fraction of the total number of proliferating cells after naphthalene injury. ${ }^{11}$ No effect was observed on the fold increase in PNEC numbers between wt and $\mathrm{Gfil}^{-1-}$ mice. Thus, whereas Gfil appears to be critical for the differentiation of the majority of PNEC, a small subset of $\mathrm{Gfil}^{-/-}$ PNEC are capable of NE differentiation. Although such PNEC have impaired proliferation, their response to postnaphthalene cues is not statistically different from PNEC in wt littermates. Importantly, the reduced proliferation seen in $G f i 1^{-1}$ NE cells is consistent with the previously proposed role for Gfil in controlling neuroendocrine cancer growth. ${ }^{31}$

Following naphthalene injury, the airways undergo dynamic regeneration characterized by reconstitution of depleted Clara cells. ${ }^{6}$ Staining of cells that have incorporated $\mathrm{BrdU}$ is representative of cell proliferation in the reconstituting lungs. We find a markedly reduced induction in the overall proliferation rate in the airway epithelium in $\mathrm{Gfil}^{-/-}$ mice after naphthalene treatment. The reduced proliferation and apoptosis of $\mathrm{Gfi1}^{-/-}$airway epithelium was associated with a profound impairment in the absolute levels of NE marker expression and BrdU + CGRP + cells. Although these results might support a previously hypothesized paracrine and growth stimulatory role of PNEC, ${ }^{7}$ we found no difference in the post-naphthalene induced rate of proliferation and apoptosis in wt $v s \mathrm{Gfil}^{-/-}$terminal airway epithelial cells. Moreover, regeneration of the $\mathrm{CC} 10+$ cells was virtually identical between wt and $\mathrm{Gfil}^{-1-}$ littermates. Thus, the data fail to argue conclusively that the NE phenotype (or Gfil) modulates the regeneration process in bronchioloalveolar junctions following naphthalene-induced injury.

As reported by others, the regeneration at bronchioloalveolar junction may be independent of PNECs. ${ }^{18,20}$ Although PNEC do not function as progenitors in the reconstitution process, ${ }^{16,17,19}$ the relevant progenitor cells have been suggested to enter a transient NE differentiation phase before differentiating to Clara cells, and even to be the potential progenitors for SCLC. ${ }^{34}$ Although the NE phenotype is central to SCLC oncogenesis, ${ }^{22,32}$ our data indicate that 

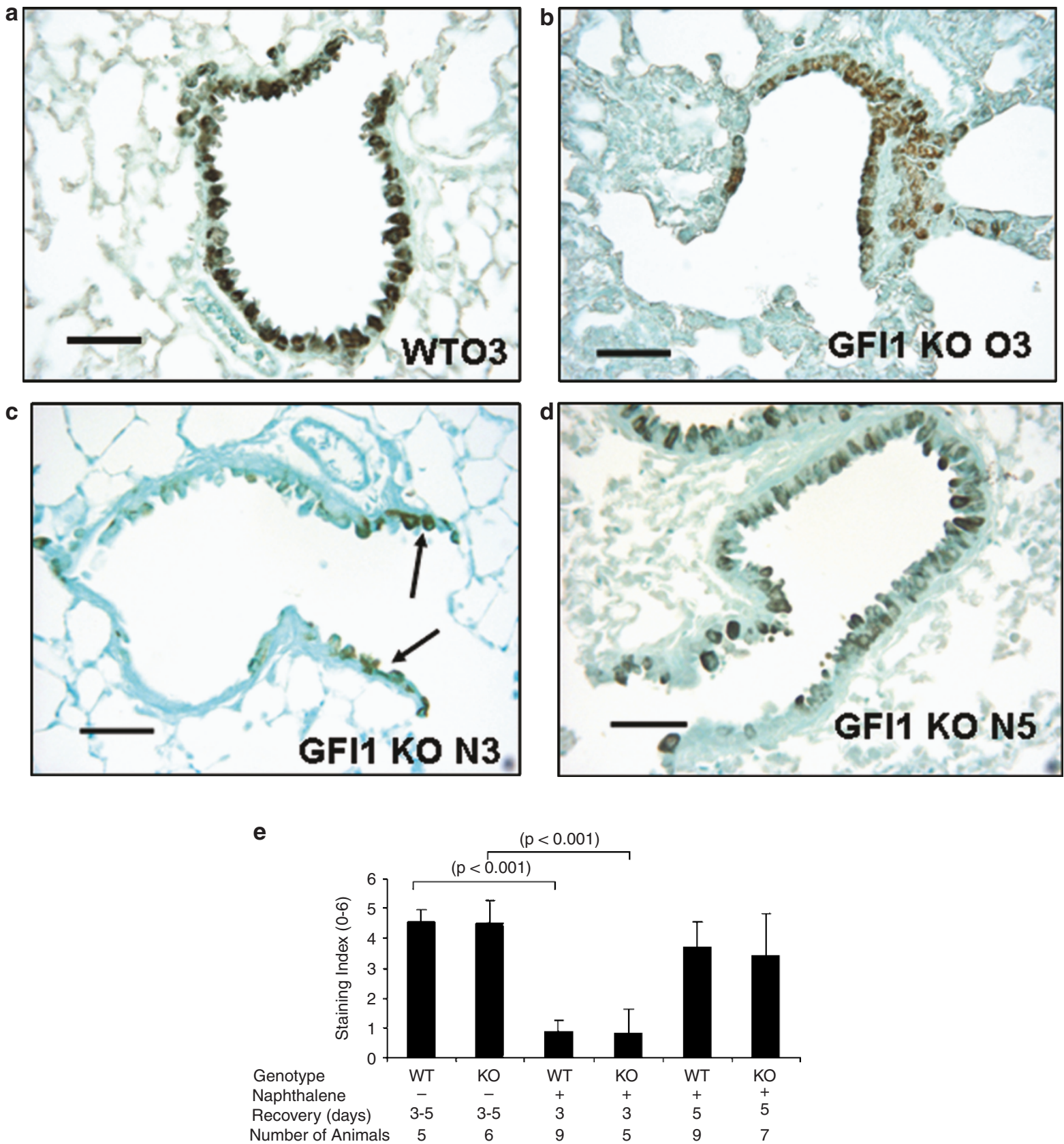

Figure 3 Clara cell lung reconstitution is efficient in $\mathrm{Gfi}^{-/-}$mice. (a and $\left.\mathbf{b}\right)$ Distribution of CC10-positive cells in the terminal airways of wtO3 and $\mathrm{Gfi}^{-1-}$ mice (GFI1 KO O3) 3 days after oil treatment (Scale bar, $50 \mu \mathrm{m}$ ). (c) CC10 immunohistochemical detection 3 days post-naphthalene administration (GFI1 KO N3) demonstrates ablation most of CC10 expressing Clara cells in $\mathrm{Gfi}^{-/-}$mice. Arrows indicate rare positive cells (Scale bar, $\left.50 \mu \mathrm{m}\right)$. (d) CC10

immunohistochemical detection in Gfi1 ${ }^{-1-}$ mice 5 days after naphthalene treatment (GFI1 KO N5) demonstrates Clara cell reconstitution in a terminal airway (Scale bar, $50 \mu \mathrm{m}$ ). (e) Quantification of the immunohistochemical data of CC10 staining of both wt and Gfi ${ }^{-1-}$ mice (KO) 3 and 5 days following oil or naphthalene administration (3-5 represent combined data for both days). Data represent the staining index ( $m e a n \pm$ standard error of mean) in terminal and small bronchioli.

it is not likely to be a driving force in the process of postnaphthalene reconstitution.

\section{ACKNOWLEDGEMENT}

This work was partially supported by NIH CA112405, and by the Intramural Research Program of the $\mathrm{NIH}$. We thank Michael Jansen PhD for assistance with statistical analyses.

\section{DUALITY OF INTEREST}

None declared.

1. Ito T. Differentiation and proliferation of pulmonary neuroendocrine cells. Prog Histochem Cytochem 1999;34:247-322.

2. Lauweryns JM, Van RL. Immunocytochemical localization of aromatic L-amino acid decarboxylase in human, rat, and mouse 
bronchopulmonary and gastrointestinal endocrine cells. J Histochem Cytochem 1988;36:1181-1186.

3. Fu XW, Nurse CA, Wong V, Cutz E. Hypoxia-induced secretion of serotonin from intact pulmonary neuroepithelial bodies in neonatal rabbit. J Physiol 2002;539:503-510.

4. Cutz E, Jackson A. Neuroepithelial bodies as airway oxygen sensors. Respir Physiol 1999;115:201-214.

5. Neuhuber WL. Lung sensors: complex functions require complex structures. Am J Respir Cell Mol Biol 2003;28:265-266.

6. Stevens TP, McBride JT, Peake JL, et al. Cell proliferation contributes to PNEC hyperplasia after acute airway injury. Am J Physiol 1997;272:L486-L493.

7. Linnoila RI. Functional facets of the pulmonary neuroendocrine system. Lab Invest 2006;86:425-444.

8. Schmeltz I, Tosk J, Hoffmann D. Formation and determination of naphthalenes in cigarette smoke. Anal Chem 1976;48:645-650.

9. Plopper CG, Macklin J, Nishio SJ, et al. Relationship of cytochrome P450 activity to Clara cell cytotoxicity. III. Morphometric comparison of changes in the epithelial populations of terminal bronchioles and lobar bronchi in mice, hamsters, and rats after parenteral administration of naphthalene. Lab Invest 1992;67:553-565.

10. Buckpitt A, Buonarati M, Avey LB, et al. Relationship of cytochrome P450 activity to Clara cell cytotoxicity. II. Comparison of stereoselectivity of naphthalene epoxidation in lung and nasal mucosa of mouse, hamster, rat and rhesus monkey. J Pharmacol Exp Ther 1992;261:364-372.

11. Reynolds SD, Giangreco A, Power JH, et al. Neuroepithelial bodies of pulmonary airways serve as a reservoir of progenitor cells capable of epithelial regeneration. Am J Pathol 2000;156:269-278.

12. Peake JL, Reynolds SD, Stripp BR, et al. Alteration of pulmonary neuroendocrine cells during epithelial repair of naphthalene-induced airway injury. Am J Pathol 2000;156:279-286.

13. Aguayo SM. Pulmonary neuroendocrine cells in tobacco-related lung disorders. Anat Rec 1993;236:122-127.

14. Johnson DE, Anderson WR, Burke BA. Pulmonary neuroendocrine cells in pediatric lung disease: alterations in airway structure in infants with bronchopulmonary dysplasia. Anat Rec 1993;236:115-119.

15. Gosney JR, Sissons MC, Allibone RO, et al. Pulmonary endocrine cells in chronic bronchitis and emphysema. J Pathol 1989;157:127-133.

16. Reynolds SD, Hong KU, Giangreco A, et al. Conditional Clara cell ablation reveals a self-renewing progenitor function of pulmonary neuroendocrine cells. Am J Physiol Lung Cell Mol Physiol 2000;278:L1256-L1263.

17. Hong KU, Reynolds SD, Giangreco A, et al. Clara cell secretory proteinexpressing cells of the airway neuroepithelial body microenvironment include a label-retaining subset and are critical for epithelial renewal after progenitor cell depletion. Am J Respir Cell Mol Biol 2001;24: $671-681$

18. Giangreco A, Reynolds SD, Stripp BR. Terminal bronchioles harbor a unique airway stem cell population that localizes to the bronchoalveolar duct junction. Am Pathol 2002;161:173-182.

19. Hong KU, Reynolds SD, Watkins $S$, et al. Basal cells are a multipotent progenitor capable of renewing the bronchial epithelium. Am J Pathol 2004;164:577-588.
20. Kim $\mathrm{CF}$, Jackson $\mathrm{EL}$, Woolfenden $\mathrm{AE}$, et al. Identification of bronchioalveolar stem cells in normal lung and lung cancer. Cell 2005:121:823-835

21. Hong KU, Reynolds SD, Watkins S, et al. In vivo differentiation potential of tracheal basal cells: evidence for multipotent and unipotent subpopulations. Am J Physiol Lung Cell Mol Physiol 2004;286:L643L649.

22. Watkins DN, Berman DM, Burkholder SG, et al. Hedgehog signalling within airway epithelial progenitors and in small-cell lung cancer. Nature 2003;422:313-317.

23. Gilks CB, Bear SE, Grimes HL, et al. Progression of interleukin-2 (IL-2)dependent rat $\mathrm{T}$ cell lymphoma lines to IL-2-independent growth following activation of a gene (Gfi-1) encoding a novel zinc finger protein. Mol Cell Biol 1993:13:1759-1768.

24. Schmidt $T$, Zornig $M$, Beneke $R$, et al. MoMuLV proviral integrations identified by Sup-F selection in tumors from infected myc/pim bitransgenic mice correlate with activation of the Gfi1gene. Nucleic Acids Res 1996;24:2528-2534.

25. Liao X, Tang Y, Chattopadhyay SK, et al. Upregulation of Gfi-1, a gene involved in IL-2-independent growth of T cells, in a murine retrovirusinduced immunodeficiency syndrome. In vivo 1997;11:9-12.

26. Scheijen B, Jonkers J, Acton D, et al. Characterization of pal-1, a common proviral insertion site in murine leukemia virus-induced lymphomas of c-myc and Pim-1 transgenic mice. J Virol 1997;71: 9-16.

27. Nolo R, Abbott LA, Bellen HJ. Senseless, a Zn finger transcription factor, is necessary and sufficient for sensory organ development in Drosophila. Cell 2000;102:349-362.

28. Wallis $D$, Hamblen $M$, Zhou $Y$, et al. The zinc finger transcription factor Gfi1, implicated in lymphomagenesis, is required for inner ear hair cell differentiation and survival. Development 2003;130: 221-232.

29. Hertzano R, Montcouquiol M, Rashi-Elkeles S, et al. Transcription profiling of inner ears from Pou4f3(ddl/ddl) identifies Gfi1 as a target of the Pou4f3 deafness gene. Hum Mol Genet 2004;13: 2143-2153.

30. Yang Z, Ding K, Pan L, et al. Math5 determines the competence state of retinal ganglion cell progenitors. Dev Biol 2003;264:240-254.

31. Kazanjian A, Wallis D, Au N, et al. Growth factor independence-1 is expressed in primary human neuroendocrine lung carcinomas and mediates the differentiation of murine pulmonary neuroendocrine cells. Cancer Res 2004;64:6874-6882.

32. Linnoila Rl, Mulshine JL, Steinberg SM, et al. Neuroendocrine differentiation in endocrine and nonendocrine lung carcinomas. Am J Clin Pathol 1988;90:641-652.

33. Jensen $\mathrm{SM}$, Jones JE, Pass $\mathrm{H}$, et al. Clara cell $10 \mathrm{kDa}$ protein mRNA in normal and atypical regions of human respiratory epithelium. Int J Cancer 1994;58:629-637.

34. Borges M, Linnoila Rl, van de Velde HJ, et al. An achaete-scute homologue essential for neuroendocrine differentiation in the lung. Nature 1997;386:852-855.

35. Stripp BR, Maxson K, Mera R, et al. Plasticity of airway cell proliferation and gene expression after acute napththalene injury. Am J Physiol 1995;269:L791-L799. 